\title{
DISTRIBUTED SCHEDULING OF SENSOR NETWORKS FOR IDENTIFICATION OF SPATIO-TEMPORAL PROCESSES
}

\author{
MACIEJ PATAN \\ Institute of Control and Computation Engineering \\ University of Zielona Góra, ul. Podgórna 50, 65-246 Zielona Góra, Poland \\ e-mail:m.pataneissi.uz.zgora.pl
}

\begin{abstract}
An approach to determine a scheduling policy for a sensor network monitoring some spatial domain in order to identify unknown parameters of a distributed system is discussed. Given a finite number of possible sites at which sensors are located, the activation schedule for scanning sensors is provided so as to maximize a criterion defined on the Fisher information matrix associated with the estimated parameters. The related combinatorial problem is relaxed through operating on the density of sensors in lieu of individual sensor positions. Then, based on the adaptation of pairwise communication algorithms and the idea of running consensus, a numerical scheme is developed which distributes the computational burden between the network nodes. As a result, a simple exchange algorithm is outlined to solve the design problem in a decentralized fashion.
\end{abstract}

Keywords: distributed-parameter system, parameter estimation, running consensus, scanning measurements, sensor network.

\section{Introduction}

Systems with spatio-temporal dynamics, commonly known as Distributed-Parameter Systems (DPSs), constitute one of the most general and important classes of systems which are widely used in modelling for a wide variety of real-world engineering problems. Recent developments in technical systems force engineers to search for more precise mathematical models of the phenomena considered, which leads directly to the description of the system at hand using Partial Differential Equations (PDEs) as the lumped descriptions often become unsatisfactory and cannot provide a sufficient approximation of the investigated system. Despite the sophisticated formulation, such models provide high quality and efficiency of simulations and control techniques.

One of the essential issues encountered while trying to design an experimental set-up for parameter estimation of a distributed system is an appropriate configuration of the monitoring system in terms of spatial deployment of sensors. The impossibility to observe the system states over the entire spatial domain implies the question of where to locate sensors and how to schedule the observations so as to accurately estimate the unknown system parameters. This question becomes especially important in the context of recent advances in distributed sensor networks
(Ögren et al., 2004; Cassandras and Li, 2005; Martínez and Bullo, 2006).

Over the past years, laborious research on the development of strategies for efficient sensor placement has been conducted (for reviews, see Kubrusly and Malebranche, 1985; Rafajłowicz, 1986; Uciński, 2000a; 2005; van de Wal and de Jager, 2001; Patan, 2004). However, most techniques communicated by various authors usually rely on exhaustive search over a predefined set of candidates and the combinatorial nature of the design problem is taken into account very occasionally (van de Wal and de Jager, 2001). Obviously, such an approach is feasible for a relatively small number of possible sensor locations and becomes useless as the number of possible location candidates increases. Although the number of sensor placement techniques developed to manage the problems of practical scale is very limited (cf. Uciński, 2005; Kubrusly and Malebranche, 1985; Patan, 2004), some effective approaches have been proposed to cover various experimental settings, including stationary (Nehorai et al., 1995; Uciński, 2000a; Patan and Patan, 2005; Point et al., 1996; Patan and Uciński, 2008; Joshi and Boyd, 2009), scanning (Patan and Uciński, 2005; Patan, 2006; 2008; Uciński and Demetriou, 2004) or moving observations (Porat and Nehorai, 1996; Jeremić and Neho- 
rai, 2000; Uciński, 2000b; Rafajłowicz, 1986; Uciński and Chen, 2005; Patan et al., 2008; Patan, 2009a; Demetriou and Hussein, 2009; Song et al., 2009; Uciński and Patan, 2010). In addition to this, almost all approaches to measurement scheduling in sensor networks reported so far rely on centralized techniques, which assume the existence of some superior entity to oversee the whole network and optimize the observation strategy. The distributed nature of the design problem is taken into account very occasionally. Recent advancements in sensor networks necessitate effective, distributed and fault-tolerant algorithms for computation and information exchange.

The main aim of the research reported here was to develop a decentralized approach to sensor scheduling which, while being independent of a particular model of the dynamic DPS in question, would be versatile enough to cope with practical monitoring networks consisting of a large number of sensors. The proposed approach substantially extends the efficient approach to distributed sensor configuration reported by Patan (2009b) to the more general setting of scanning sensor networks, where the observation system comprises multiple stationary sensors located at already specified locations and it is desired to activate only a subset of them during a given time interval while the other sensors remain dormant (Demetriou, 2000). A reason for not using all the available sensors could be the reduction of the observation system complexity and/or the cost of operation and maintenance (van de Wal and de Jager, 2001). Selecting the best subset of sensors to activate constitutes an inherently discrete large-scale resource allocation problem whose solution may be prohibitively time-consuming. An effective approach to overcome those difficulties, based on the branch and bound technique dedicated to such a kind of optimization tasks, has been proposed by Uciński and Patan (2007) in the context of stationary sensors and further refined for different experimental settings (Patan and Uciński, 2008; 2010a; 2010b). Although a parallelization of calculations over network nodes is also possible (Patan, 2008), it is based on centralized algorithms, since the full distribution of computations is highly non-trivial and requires relatively high computational resources per each node.

As an alternative approach suitable for situations where the numbers of candidate and gauged sites are rather large, a method whose idea is to operate on sensor densities per unit area instead of the individual sensor positions is presented. This convenient reformulation makes it possible to apply some powerful tools of convex analysis. The basic idea is to adopt the results for the socalled clusterization-free designs set forth by Uciński and Patan (2002). These, in turn, are reminiscent of the idea of replication-free designs which have emerged relatively late in the context of spatial statistics (see Fedorov and Hackl, 1997; Müller, 2007). The decided advantage of such an approach is that it can be relatively easily tailo- red to the framework of the so-called gossip algorithms, in which each node communicates with no more than one neighbor at each time instant (Xiao and Boyd, 2004; Boyd et al., 2006). The different nodes of the network independently calculate and store the desired quantities and their final estimates are obtained via a consensus between the network nodes in a fully decentralized fashion. The advantages of this scheme of information exchange seem to be clear as no powerful information fusion center is necessary and it is robust with respect to individual sensor faults as the global estimate is recorded at all sensor nodes and can easily be recovered. Moreover, it leads to very simple implementation and efficient performance of the resulting procedures.

\section{Optimal measurement strategy}

2.1. System description. Consider a bounded spatial domain $\Omega \subset \mathbb{R}^{d}$ with a sufficiently smooth boundary $\Gamma$, a bounded time interval $T=\left(0, t_{f}\right]$, and a distributed parameter system whose scalar state $y$ at a spatial point $x \in \bar{\Omega} \subset \mathbb{R}^{d}$ and a time instant $t \in \bar{T}$ is governed by the partial differential equation

$$
\frac{\partial y}{\partial t}=\mathcal{F}(x, t, y, \theta) \quad \text { in } \Omega \times T,
$$

where $\mathcal{F}$ is a well-posed, possibly nonlinear, differential operator which involves first- and second-order spatial derivatives and may include terms accounting for forcing inputs specified a priori, with $\theta$ being an $m$-dimensional vector of unknown constant parameters which must be estimated using observations of the system. The PDE (1) is accompanied by the appropriate boundary and initial conditions

$$
\begin{aligned}
\mathcal{B}(x, t, y, \theta) & =0 & & \text { on } \Gamma \times T, \\
y & =y_{0} & & \text { in } \Omega \times\{t=0\},
\end{aligned}
$$

respectively, $\mathcal{B}$ being an operator acting on the boundary $\Gamma$ and $y_{0}=y_{0}(x)$ a given function. We assume that the forms of $\mathcal{F}$ and $\mathcal{B}$ are given explicitly up to vector $\theta$.

Let us assume that the state $y$ is observed directly (an extension to a more general situation where the state is observed indirectly can be provided without serious difficulties, see the work of Patan (2004) for details) by $N$ pointwise sensors, from among which only $n$ are activated at time instants $0<t_{0}<t_{1}<\cdots<t_{K}=t_{f}$ and will gather the continuous measurements for the duration of each subinterval $T_{k}=\left(t_{k-1}, t_{k}\right], k=1, \ldots, K$. Forming such an arbitrary partition on the time interval $T$, the 'scanning' observation strategy considered can be formally represented as

$$
\begin{gathered}
z^{\ell}(t)=y\left(x_{k}^{\ell}, t ; \theta\right)+\varepsilon\left(x_{k}^{\ell}, t\right), \quad t \in T_{k}, \\
\ell=1, \ldots, n, \quad k=1, \ldots K
\end{gathered}
$$


where $z^{\ell}(t)$ is the scalar output and $x_{k}^{\ell} \in X$ stands for the location of the $\ell$-th sensor at time subinterval $T_{k}, X$ signifies the part of the spatial domain $\Omega$ where the measurements can be made and $\varepsilon\left(x_{k}^{\ell}, t\right)$ denotes the measurement noise, which is customarily assumed to be zeromean, Gaussian, spatial uncorrelated and white (Quereshi et al., 1980; Amouroux and Babary, 1988), i.e.,

$$
\mathrm{E}\left\{\varepsilon\left(x^{\ell}, t\right) \varepsilon\left(x^{\ell^{\prime}}, t^{\prime}\right)\right\}=\sigma^{2} \delta_{\ell \ell^{\prime}} \delta\left(t-t^{\prime}\right),
$$

where $\sigma^{2}$ defines the intensity of the noise, $\delta_{i j}$ and $\delta(\cdot)$ standing for the Kronecker and Dirac delta functions, respectively. Although white noise is a physically impossible process, it constitutes a reasonable approximation to a disturbance whose adjacent samples are uncorrelated at all time instants for which the time increment exceeds some value which is small compared with the time constants of the DPS.

2.2. Estimation accuracy measure. Given the model (1)-(3) and the outcomes of the measurements $z^{\ell}(\cdot)$, $\ell=1, \ldots, n$ on time intervals $T_{k}$, estimate $\theta$ by $\widehat{\theta}$, a global minimizer of the output least-squares criterion

$$
\mathcal{J}(\vartheta)=\sum_{k=1}^{K} \sum_{\ell=1}^{n} \frac{1}{t_{f}} \int_{T_{k}}\left\{z^{\ell}(t)-y\left(x_{k}^{\ell}, t ; \vartheta\right)\right\}^{2} \mathrm{~d} t,
$$

where $y(\cdot, \cdot ; \vartheta)$ denotes the solution to (1)-(3) for a given value of the parameter vector $\vartheta$.

Inevitably, the covariance matrix $\operatorname{cov}(\widehat{\theta})$ of the above least-squares estimator depends on the active sensor locations $x_{k}^{\ell}$. This fact suggests that we may attempt to select them so as to yield the best estimates of the system parameters. To form a basis for a comparison of different locations, a quantitative measure of the 'goodness' of particular sensor configurations is required. Such a measure $\Psi$ is customarily based on the concept of the Fisher Information Matrix (FIM), which is widely used in optimum experimental design theory for lumped systems (Fedorov and Hackl, 1997; Atkinson et al., 2007). In our setting, owing to the character of noise in (4), the FIM is given by (Quereshi et al., 1980)

$$
M=\sum_{k=1}^{K} \sum_{\ell=1}^{n} \frac{1}{t_{f}} \int_{T_{k}} g\left(x_{k}^{\ell}, t\right) g^{\top}\left(x_{k}^{\ell}, t\right) \mathrm{d} t,
$$

where

$$
g(x, t)=\left[\begin{array}{lll}
\frac{\partial y(x, t ; \vartheta)}{\partial \vartheta_{1}}, & \ldots, & \frac{\partial y(x, t ; \vartheta)}{\partial \vartheta_{m}}
\end{array}\right]_{\vartheta=\theta^{0}}^{\top}
$$

stands for the so-called sensitivity vector. Since in the nonlinear case $g$ depends on the estimated parameters, some preliminary estimate $\theta^{0}$ is required for its calculation. Usually some known nominal values of the parameters $\theta$ can be used or we can apply estimates obtained in previous experiments (Uciński, 2005; Sun, 1994; Rafajłowicz, 1983). Up to a constant scalar multiplier, the inverse of the FIM constitutes a good approximation of $\operatorname{cov}(\widehat{\theta})$ provided that the time horizon is large, the nonlinearity of the model with respect to its parameters is mild, and the measurement errors are independently distributed and have small magnitudes (Fedorov and Hackl, 1997).

As for a specific form of $\Psi$, various options exist (Fedorov and Hackl, 1997; Atkinson et al., 2007), but the most popular criteria to be minimized include the following:

- the D-optimality (determinant) criterion,

$$
\Psi(M)=-\log \operatorname{det}(M)
$$

- the A-optimality (trace) criterion,

$$
\Psi(M)=\operatorname{tr}(M) .
$$

The resulting D- and A-optimum sensor configurations lead, respectively, to the minimum volume and minimum mean of axes of the uncertainty ellipsoid for the parameter estimates.

2.3. Sensor scheduling problem. The optimal sensor scheduling problem consists in seeking for each time subinterval $T_{k}$ the best subset of $n$ locations from among $N$ given potential ones. More precisely, the problem is to divide for each time subinterval the $N$ available sensor nodes into $n$ active ones and the remaining $N-n$ dormant ones so as to maximize the criterion (10) associated with the parameters to be estimated. Introducing for each possible location $x^{i}(i=1, \ldots, N)$ a set of variables $v_{k}^{i}$, each of them taking the value 1 or 0 depending on whether or not a sensor residing at $x^{i}$ is activated during $T_{k}$, the FIM in (7) can then be rewritten as

$$
M\left(v_{1}, \ldots, v_{K}\right)=\sum_{i=1}^{N} \sum_{k=1}^{K} v_{k}^{i} M_{k}\left(x^{i}\right),
$$

where

$$
M_{k}\left(x^{i}\right)=\frac{1}{t_{f}} \int_{T_{k}} g\left(x^{i}, t\right) g^{\top}\left(x^{i}, t\right) \mathrm{d} t
$$

and $v^{i}=\left(v_{1}^{i}, \ldots, v_{K}^{i}\right)$. It is straightforward to verify that the matrices $M_{k}\left(x^{i}\right)$ are nonnegative definite and, therefore, so is $M\left(v_{1}, \ldots, v_{K}\right)$. Hence, our design problem takes the following form.

Problem 1. Find a sequence $v=\left(v^{1}, \ldots, v^{N}\right)$ to maximize

$$
\mathcal{P}(v)=\Psi(M(v))
$$


subject to

$$
\begin{aligned}
& \sum_{i=1}^{N} v_{k}^{i}=n, \quad k=1, \ldots, K, \\
& v_{k}^{i}=0 \text { or } 1, \quad i=1, \ldots, N, \quad k=1, \ldots, K .
\end{aligned}
$$

This constitutes a $0-1$ integer programming problem which necessitates smart and efficient solution. A key difficulty in developing successful numerical techniques for such a discrete optimization task is that the number of sensor nodes in the network may be quite large, which considerably increases the complexity of the problem. Another important issue is to properly decompose a computational task in order to provide a possibility for a decentralized procedure for determining an optimal sensor activation schedule.

Patan (2004) and Uciński (2005) proposed a general sequential computational scheme to solve a similar problem based on the notion of the so-called directly constrained design measures. A brief characterization of the main ideas underlying such an approach and the development of the appropriate decentralized numerical algorithm will constitute the remainder of the paper.

\section{Approximate solution via continuous relaxation}

3.1. Problem of optimal sensor densities. When the number of candidate sites and sensors to be activated $n$ is large, we can operate on the spatial density of sensors (i.e., the number of sensors per unit area), rather than on the individual sensor locations. In order to get rid of the combinatorial nature of the original problem, we relax the definition of the set of admissible solutions by making use of the observation that the density of sensors over the subinterval $T_{k}$ can be approximately described by a probability measure $\xi_{k}(\mathrm{~d} x)$ on the space $(X, \mathcal{B})$, where $\mathcal{B}$ is the $\sigma$-algebra of all Borel subsets of $X$. Such an extension of the set of feasible solutions makes it possible to apply convenient and efficient mathematical tools of convex programming theory. As for practical interpretation of the so produced results, it is, e.g., to partition $X$ into non-overlapping subdomains $X_{i}$ of relatively small areas and then, on each subinterval $T_{k}$, to allocate to each of them the number

$$
N_{r}^{i}=\left\lceil N \int_{X_{i}} \xi_{k}(\mathrm{~d} x)\right\rceil
$$

of sensors (here $\lceil\rho\rceil$ is the smallest integer greater than or equal to $\rho$ ).

Accordingly, our aim is to find probability measures $\xi_{k}, k=1, \ldots, K$ over $X$ which are absolutely continuous with respect to the Lebesgue measure and satisfy by definition the conditions $\int_{X} \xi_{k}(\mathrm{~d} x)=1, \quad k=1, \ldots, K$. The integration above is to be understood in the LebesgueStieltjes sense. In what follows we briefly write

$$
\xi=\left(\xi_{1}, \ldots, \xi_{K}\right)
$$

and call $\xi$ a design measure.

Consequently, we replace (7) by

$$
M(\xi)=\sum_{k=1}^{K} \int_{X} M_{k}(x) \xi_{k}(\mathrm{~d} x) .
$$

This leads directly to the so-called continuous designs, which constitute the basis of the modern theory of optimal experiments (Fedorov and Hackl, 1997; Patan, 2004; Uciński, 2005).

Furthermore, another natural assumption is that the densities of sensor allocation are bounded by some prescribed level, i.e.,

$$
\xi_{k}(\mathrm{~d} x) \leq \omega(\mathrm{d} x), \quad k=1, \ldots, K,
$$

where $\omega(\mathrm{d} x)$ can be interpreted in terms of some reasonable level related to the maximal possible 'number' of sensors per d $x$ (Fedorov and Hackl, 1997; Patan, 2004; Uciński, 2005) such that $\int_{X} \omega(\mathrm{d} x) \geq 1$. Finally, we come up with the following optimization problem:

Problem 2. Find a design measure $\xi \in \Xi(X), \Xi(X)$ being the set of all $K$-tuples defined by (17), to maximize

$$
\mathcal{P}(\xi)=\Psi(M(\xi))
$$

subject to

$$
\xi_{k}(\mathrm{~d} x) \leq \omega(\mathrm{d} x), \quad k=1, \ldots, K .
$$

The design $\xi^{\star}$, being the solution to the problem above, is then said to be a $(\Psi, \omega)$-optimal design (Fedorov and Hackl, 1997).

3.2. Characterization of the optimal solutions. In order to derive an efficient decentralized algorithm for solving Problem 2, a number of important properties of the optimal design $\xi^{\star}$ can be provided from the general results of Patan (2004) and Uciński (2005). In the remainder of this paper we shall make the following assumptions:

(A1) $X$ is compact.

(A2) $g \in C\left(X \times T ; \mathbb{R}^{m}\right)$.

(A3) $\Psi$ is convex.

(A4) If $M_{1} \preceq M_{2}$, then $\Psi\left(M_{1}\right) \geq \Psi\left(M_{2}\right)$ is convex.

(A5) $\omega(\mathrm{d} x)$ is atomless, i.e., for any set $\Delta X \subset X$ with some positive measure there exists its subset $\Delta X^{\prime} \subset$ $\Delta X$ with positive measure such that

$$
\int_{\Delta X^{\prime}} \omega(\mathrm{d} x)<\int_{\Delta X} \omega(\mathrm{d} x) .
$$


(A6) There exists a finite real value $q$ such that $\widetilde{\Xi}(X)=$ $\{\xi: \xi$ satisfy (21) and $\mathcal{P}(\xi) \leq q\} \neq \emptyset$.

(A7) For any $\xi \in \widetilde{\Xi}(X)$ and $\bar{\xi} \in \Xi(X), \Psi$ is Fréchet differentiable at $M(\xi)$ in the direction of $M(\xi-\bar{\xi})$.

Assumptions (A1) (A4) are quite natural, since they determine a regularity of solution and allows us to stay within the framework of convex analysis, which greatly facilitates subsequent deliberations. Assumption (A5) establishes a required level of design relaxation allowing application of techniques for continuous optimization. As for (A6) it only states that there exist designs with finite values of $\Psi$, which constitutes a rather mild and quite logical requirement. Finally, Assumption (A7) simply amounts to the existence of the directional derivative whose form must be on the one hand specific, but on the other for most practical criteria such a condition is not particularly restrictive. Requiring $\Psi$ to be differentiable with respect to the individual elements of its matrix argument, we obtain

$$
\psi_{k}(x, \xi)=\varsigma(\xi)-\phi_{k}(x, \xi)
$$

where

$$
\begin{aligned}
\phi_{k}(x, \xi) & =-\operatorname{tr}\left[\stackrel{\circ}{\Psi}[M(\xi)] M_{k}(x)\right], \\
\varsigma(\xi) & =-\frac{1}{K} \operatorname{tr}[\stackrel{\circ}{\Psi}[M(\xi)] M(\xi)],
\end{aligned}
$$

and

$$
\stackrel{\circ}{\Psi}[M(\xi)]=\left.\frac{\partial \Psi(M)}{\partial M}\right|_{M=M(\xi)} .
$$

For particular criteria we have, e.g.,

- D-optimality criterion: $\stackrel{\circ}{\Psi}[M(\xi)]=M(\xi)^{-1}$,

- A-optimality criterion: $\stackrel{\circ}{\Psi}[M(\xi)]=M(\xi)^{-2}$.

In what follows, we write $\bar{\Xi}(X)$ for the collection of all the design measures whose components satisfy

$$
\xi_{k}(\Delta X)= \begin{cases}\omega(\Delta X) & \text { for } \Delta X \subset \operatorname{supp} \xi_{k}, \\ 0 & \text { for } \Delta X \subset X \backslash \operatorname{supp} \xi_{k},\end{cases}
$$

where the support of a measure $\xi$ is defined as the closed set $\operatorname{supp} \xi=X \backslash \bigcup\{G: \xi(G)=0, G$ is open $\}$, (cf. Rao, 1987 , p.80). Further, given a design $\xi$, we will say that the function $\psi_{k}(\cdot, \xi)$ defined by (23) separates sets $X_{1}$ and $X_{2}$ with respect to $\omega(\mathrm{d} x)$ if for any two sets $\Delta X_{1} \subset X_{1}$ and $\Delta X_{2} \subset X_{2}$ with equal non-zero $\omega$-measures we have

$$
\int_{\Delta X_{1}} \psi_{k}(x, \xi) \omega(\mathrm{d} x) \geq \int_{\Delta X_{2}} \psi_{k}(x, \xi) \omega(\mathrm{d} x) .
$$

Now we are able to formulate the following properties of $(\Psi, \omega)$-optimal designs, (for details, see Patan, 2004; Uciński, 2005).
Theorem 1. Let Assumptions (A1) (A7) hold. Then

(i) there exists an optimal design $\xi^{\star} \in \bar{\Xi}(X)$, and

(ii) a necessary and sufficient condition for $\xi^{\star}=$ $\left(\xi_{1}^{\star}, \ldots, \xi_{K}^{\star}\right) \in \Xi(X) \cap \widetilde{\Xi}(X)$ to be $(\Psi, \omega)$-optimal is that functions $\phi_{k}\left(\cdot, \xi^{\star}\right)$ separate $X_{r}^{\star}=\operatorname{supp} \xi_{k}^{\star}$ and its complement $X \backslash X_{k}^{\star}$ for $k=1, \ldots, K$.

From a practical point of view, the above result means that at all the support points of an optimal design $\xi^{\star}$ the mapping $\phi_{k}\left(\cdot, \xi^{\star}\right)$ should be greater than anywhere else, i.e., preferably $\operatorname{supp} \xi_{k}^{\star}$ should coincide with maximum points of $\phi_{k}\left(\cdot, \xi^{\star}\right)$. In practice, this amounts to allocating observations to the points at which we know least of all about the system response.

\section{Decentralized exchange algorithm}

4.1. Distributed data exchange. Clearly, unless the design problem considered is quite simple, we must employ a numerical algorithm to make the outlined idea useful. Moreover, the key property of the resulting procedure should be an effective distribution of computations between the sensor nodes in a fully decentralized way. In the following we assume the asynchronous time model for the configuration process. Let $r=0,1,2, \ldots$ be the discrete time index, which partitions the continuous configuration time axis into time slots $Z_{r}=\left(z_{r-1}, z_{r}\right]$.

Owing to Theorem $1, \xi_{k}^{\star}(\mathrm{d} x)$ should be nonzero in the areas where $\phi_{k}\left(\cdot, \xi^{\star}\right)$ takes on larger values. Thus the central idea when constructing a computational algorithm for sensor density optimization is to move some measure from areas with smaller values of $\phi_{k}\left(\cdot, \xi^{(r)}\right)$ to those with larger values, as we expect that such a procedure will improve $\xi^{(r)}$. A fundamental question related to this issue is whether the functions $\phi_{k}\left(\cdot, \xi^{(r)}\right)$ can be calculated or estimated in a decentralized way. From (24) we see that the only component of $\phi_{k}\left(\cdot, \xi^{(r)}\right)$ which cannot be calculated independently of other nodes is the global information matrix $M\left(\xi^{(r)}\right)$. Furthermore, from (11) it is clearly seen that this matrix is a weighted average of the local information matrices given by (12). In such a way, our task is closely related to the problem of distributed averaging on a sensor network which appears in many applications and has been a subject of extensive studies (Kempe et al., 2003; Xiao and Boyd, 2004; Boyd et al., 2006; Braca et al., 2008).

One of the simplest and popular techniques of distributed averaging is a pairwise communication flooding, also known as a gossip scheme, which in its classic version assumes that at the $r$-th time slot the $i$-th sensor contacts some neighboring node $j$ with probability $P_{i j}$, i.e., a pair $(i-j)$ is randomly and independently selected. $P$ is a doubly stochastic matrix with nonnegative entries and $P_{i j}>0$ only if node $i$ is within a range of node $j$. At this time, both nodes set their values equal to the average of 
their current values. Denoting by $M^{\ell}\left(\xi^{(r)}\right)$ an estimate of the global FIM maintained by the $\ell$-th sensor at time slot $Z_{r}$, we can write ( $\leftarrow$ is an update operator)

$$
M^{\ell}\left(\xi^{(r)}\right) \leftarrow \frac{1}{2}\left(M^{i}\left(\xi^{(r)}\right)+M^{j}\left(\xi^{(r)}\right)\right), \quad \ell \in\{i, j\} .
$$

Under some assumptions on the communication scheme, such an update leads to the mean of local FIMs of all network nodes (see the work of Xiao and Boyd (2004) for detailed conditions for this to happen).

However, in our setting the distributed averaging problem is not typical as not all of the nodes contribute to the global FIM at the $r$-th configuration slot but only those located at the support of the current design. Therefore, apart from updating local estimates of the FIM, the sensor nodes should be equipped with a mechanism to store and change the global design $\xi^{(r)}$. This can be achieved by the exchange of tokens representing the activation of the sensors at given subintervals $T_{k}$. Such tokens are transferred between nodes in the situation where a neighbor node over a particular observation subinterval $T_{k}$ is more informative in the sense of the function $\phi_{k}\left(\cdot, \xi^{(r)}\right)$ calculated for the current estimate of the FIM (and, obviously, it is not activated yet). A decided advantage of such an approach is that the structure of the design $\xi^{(r)}$ is distributed via tokens over the whole network. Therefore, it is necessary to store at the $i$-th node only the binary vector $v^{i}=\left(v_{1}^{i}, \ldots, v_{K}^{i}\right)$ indicating whether or not the node owns the token at the $k$-th time instant not.

It is clear that, in order to achieve convergence to the mean of the FIMs for selected sensor nodes on a given time subinterval, only the nodes possessing tokens should follow the update (28). More precisely, we have to apply a weighted average

$$
M^{\ell}\left(\xi^{(r)}\right) \leftarrow \sum_{k=1}^{K} M^{\ell}\left(\xi_{k}^{(r)}\right), \quad \ell \in\{i, j\},
$$

where, for $v_{k}^{i}+v_{k}^{j}>0$

$$
M^{\ell}\left(\xi_{k}^{(r)}\right)=\frac{1}{\left(v_{k}^{i}+v_{k}^{j}\right)}\left(v_{k}^{i} M^{i}\left(\xi_{k}^{(r)}\right)+v_{k}^{j} M^{j}\left(\xi_{k}^{(r)}\right)\right) \text {. }
$$

The convergence of this averaging scheme with high probability is proven to be of exponential rate, (Boyd et al., 2006, Thm. 3), which is crucial for the effectiveness of the optimization task. However, there are a number of drawbacks of such an update of the FIM. First of all, since the weights $v_{k}^{i}$ are binary variables, from (30) it immediately follows that the information can flow only in one direction, i.e., from the active nodes, which own some tokens, to the dormant ones, without tokens. A direct consequence of the fact that dormant nodes cannot share the information with active ones is that for any time subinterval $T_{k}$ the nodes owning the tokens have to form a connected graph in order to achieve consensus in the network.
In practice, this may not be the case, especially when the range of each node is very limited. Furthermore, the network is not robust with respect to individual sensor faults and the network topology becomes of crucial importance.

To overcome those difficulties, instead of (30), as a generalization, the following two stage update is proposed with simultaneous optimization and data exchange:

- At the first stage of communication the nodes $i$ and $j$ estimate the global FIM according to the classical averaging scheme (28). Then, based on this estimate, the nodes calculate the function $\phi_{k}(\cdot, \cdot)$ for each observation subinterval $T_{k}$. Finally, using $\phi_{k}$ as a criterium for choosing more informative node at each $T_{k}$, the tokens can be exchanged between the nodes.

- At the second stage, if a network node owns a token for some of subintervals $T_{k}$, it is allowed to additionally contribute to its own estimate of the FIM according to the following weighted average:

$$
\begin{aligned}
M^{i}\left(\xi^{(r)}\right) & \leftarrow \frac{r-1}{r} M^{i}\left(\xi^{(r)}\right) \\
& +\frac{1}{r}\left(\sum_{k=1}^{K} v_{k}^{i}\left(M_{k}\left(x^{i}\right)+M^{i}\left(\xi_{k}^{(r)}\right)\right)\right) .
\end{aligned}
$$

The first term in (31) enforces consensus among the nodes (represents the average information from the rest of network), while the second one accounts for the increase in the total contribution of the active node at subinterval $T_{k}$, i.e., for $v_{k}^{i}=1$. The idea is quite similar to the so-called running consensus averaging (cf. Braca et al., 2008), where the sensing and the averaging stages are simultaneous and the network continues collecting data while adaptively computing the distributed estimator. Therefore, convergence in first and second moments is a straightforward generalization of the results of Braca et al. (2008).

Remark 1. The convergence rate of the proposed scheme is proportional to $r^{-1}$ (Braca et al., 2008), and this seems to be disadvantageous compared with the exponential convergence of the classic consensus scheme. However, one consequence is that such a scheme is far less sensitive with respect to specific properties of network connectivity and changes in topology than the classical gossip update. In fact, since all the nodes share and exchange information, the network is more robust to individual sensor failure. Furthermore, we can achieve a simultaneous exchange of the design measure and a proper estimation of the global information matrix without the necessity of separating these processes.

Remark 2. From a practical point of view, it is very useful to approximate the last term of (31) with an average information matrix per observational subintervals, i.e.,

$$
M^{i}\left(\xi_{k}^{(r)}\right) \approx \frac{1}{K} M^{i}\left(\xi^{(r)}\right) .
$$




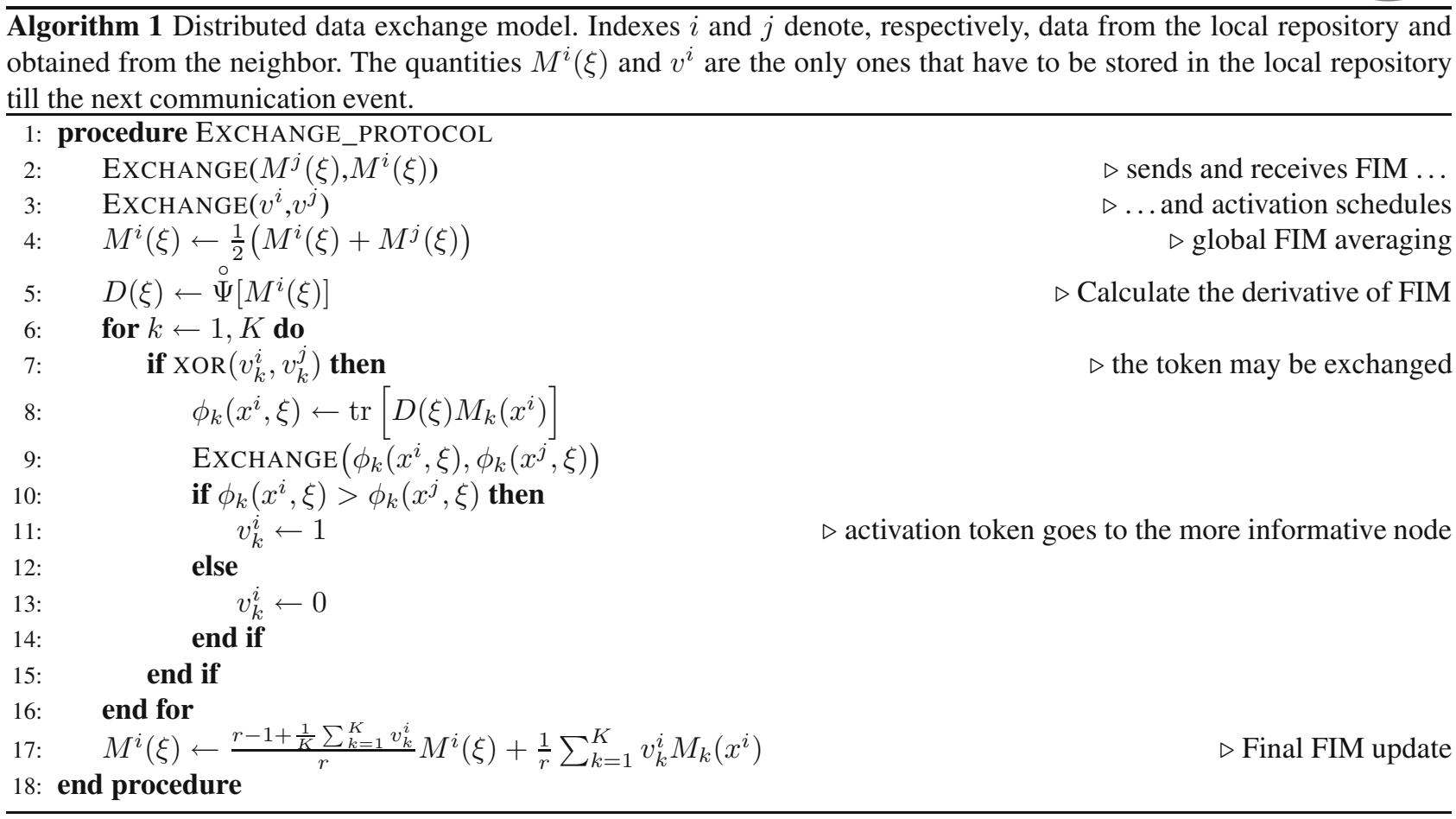

Thus, instead of 31 we have

$$
\begin{aligned}
M^{i}\left(\xi^{(r)}\right) \leftarrow & \frac{1}{r}\left(r-1+\frac{1}{K} \sum_{k=1}^{K} v_{k}^{i}\right) M^{i}\left(\xi^{(r)}\right) \\
& +\frac{1}{r}\left(\sum_{k=1}^{K} v_{k}^{i} M_{k}\left(x^{i}\right)\right) .
\end{aligned}
$$

Obviously, in such a manner a bias is introduced to the estimator, but since the corrected term is weighted with the reciprocal of $r$, it is asymptotically unbiased. The advantage is that at each node only the estimate of the total FIM has to be stored and updated instead of $K$ information matrices for each time subinterval separately.

4.2. Distributed numerical scheme. The detailed scheme of the pairwise communication process is embodied by Algorithm 1 The EXCHANGE operator involved in implementation of Algorithm 1 is responsible for duplex data exchange between two nodes, i.e., sending and receiving data to/from a connected neighbor (order depending on who initiated communication). At $r=0$ each network node starts with a global FIM estimate $M^{i}\left(\xi^{(0)}\right)$ initialized with its local information matrix $M\left(x^{i}\right)=$ $n \sum_{k=1}^{K} M_{k}\left(x^{i}\right)$ and randomly allocated tokens satisfying (14). Then at each time slot $Z_{k}$ an appropriate pair of nodes exchanges information according to Algorithm 1 .

The resulting procedure may be considered a distributed counterpart of the class of sequential exchange algorithms based on restricted design measures (cf. Uciński and Patan, 2002; Patan, 2004; Uciński, 2005) originated from experimental design theory. In particular, in the framework considered, we have $\omega(\mathrm{d} x)=\varrho \mathrm{d} x$, where $\varrho$ is a sensor density and every grid element must not contain more than one supporting point.

The algorithm performs extremely fast due to its simplicity and the central idea of operating on the sensor densities, which allows avoiding the inherent impediments related to the combinatorial sensor selection problems. As regards the convergence to an optimal design, it is assured only for a decreasing value of the exchanged design measure (Uciński, 2005), which is not the case here. Therefore, after an initial phase of fast convergence, some oscillations in $\mathcal{P}\left(\xi^{(r)}\right)$ may sometimes be observed when approaching the vicinity of the optimum. A denser spatial grid usually constitutes a remedy for this predicament (Patan, 2004; Uciński, 2005; Müller, 2007) and assures that the lack in optimality is negligible.

Another important issue is the choice of a proper communication scheme in terms of symmetric probability matrix $P$, which significantly influences the convergence rate. This is of special importance when one step update (30) can be applied for distributed averaging. In general, under some assumptions on the network connectivity graph, a suitable gossip algorithm can be provided with an exponential convergence rate. In particular, for an asynchronous time model, finding the fastest averaging algorithm is equivalent to solving the following problem (Boyd et al., 2006).

Problem 3 Find $P$ to minimize $\lambda_{2}\left(\left(1-\frac{1}{n}\right) I+\frac{1}{n} P\right)$ sub- 
ject to

$$
\sum_{j=1}^{n} P_{i j}=1, \quad i=1,2, \ldots, n, \quad P_{i j} \geq 0,
$$

where $\lambda_{2}(\cdot)$ is the second largest eigenvalue of its argument.

This problem can be cast in the framework of semidefinite programming and effectively solved in the centralized fashion. For a complete network connectivity graph, it is easy to show that the solution to Problem 3 is $P$ with all diagonal entries equal to zero and all off-diagonal entries equal to the value of $1 /(n-1)$. Since a detailed discussion on such important issues is far beyond the scope of this work, we refer the reader to the seminal papers of Xiao and Boyd (2004), as well as Boyd et al. (2006), where also a distributed subgradient scheme is provided to solve this problem in a fully decentralized way.

\section{Simulation example}

As an illustrative example of the presented approach, consider the problem of sensor configuration for parameter estimation in the process of air pollutant transport over a given urban area $\Omega$, which is a square with a side of length $1 \mathrm{~km}$. In this domain, two active sources of pollution are present, which yields the pollutant spatial concentration $y=y(x, t)$. The evolution of $y$ over the observation interval $T=(0,1000]$ (in seconds) is described by the following advection-diffusion-reaction equation:

$$
\begin{aligned}
\frac{\partial y(x, t)}{\partial t} & +\nabla \cdot(v(x, t) y(x, t))+\alpha y(x, t) \\
& =\nabla \cdot(\kappa \nabla y(x, t))+f_{1}(x)+f_{2}(x), \quad x \in \Omega,
\end{aligned}
$$

subject to the boundary and initial conditions

$$
\begin{array}{ll}
\frac{\partial y(x, t)}{\partial n}=0 & \text { on } \partial \Omega \times T, \\
y(x, 0)=y_{0} & \text { in } \Omega,
\end{array}
$$

where terms $f_{\ell}(x)=\mu_{\ell} \exp \left(-100\left\|x-\chi^{\ell}\right\|^{2}\right), \ell=1,2$ represent the pollutant sources with emission intensities $\mu_{\ell}$ located at the points $\chi^{\ell}=\left(\chi_{1}^{\ell}, \chi_{2}^{\ell}\right), \quad \ell=1,2$, and $\partial y / \partial n$ stands for the derivative of $y$ in the direction of the outward normal to the boundary $\partial \Omega$. The average spatiotemporal changes in the wind velocity field over $\Omega$ were approximated according to the model (scaled in $[\mathrm{km} / \mathrm{h}]$ )

$$
v(x, t)=7.2 \cdot\left(x_{1}+x_{2}-t \cdot 10^{-3},\left(2 x_{1}-1\right) t \cdot 10^{-3}+x_{2}-1\right) .
$$

Furthermore, $\kappa$ denotes an unknown turbulent diffusion coefficient and $\alpha=0.02 \mathrm{~s}^{-1}$ stands for the absorption rate modelling a slow decay of the pollutant. Figure 1 illustrates the resulting complex process dynamics.
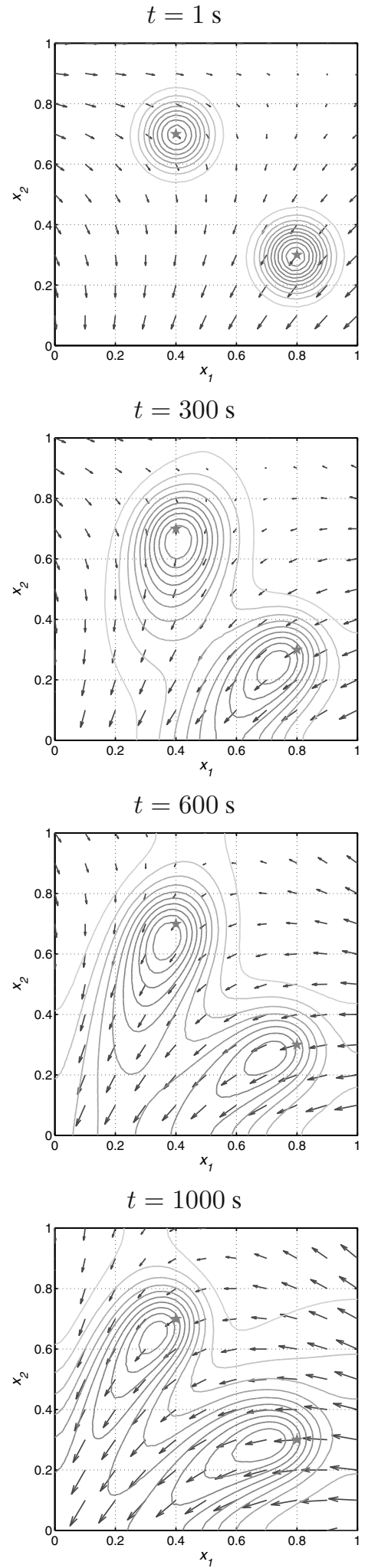

Fig. 1. Temporal changes in the wind velocity field and pollutant concentration (stars indicate the locations of pollutant sources). 


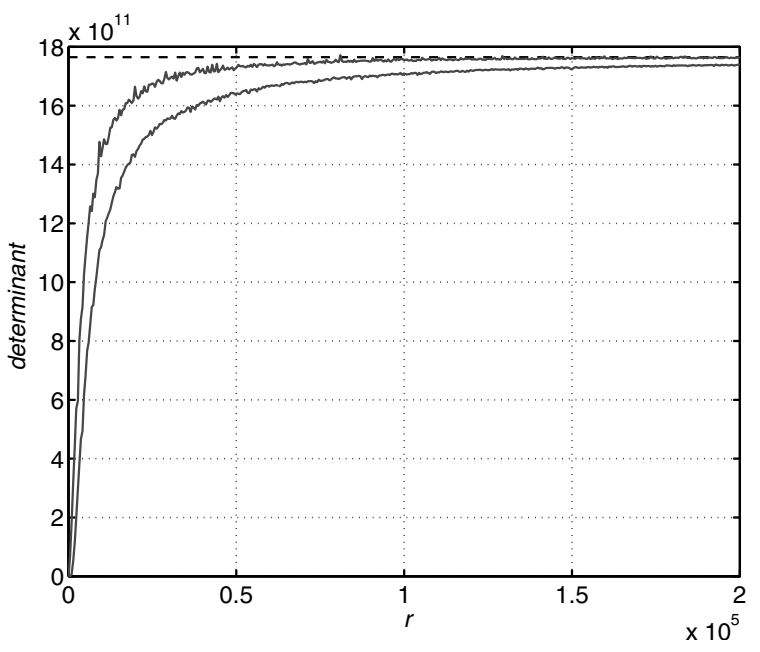

(a)

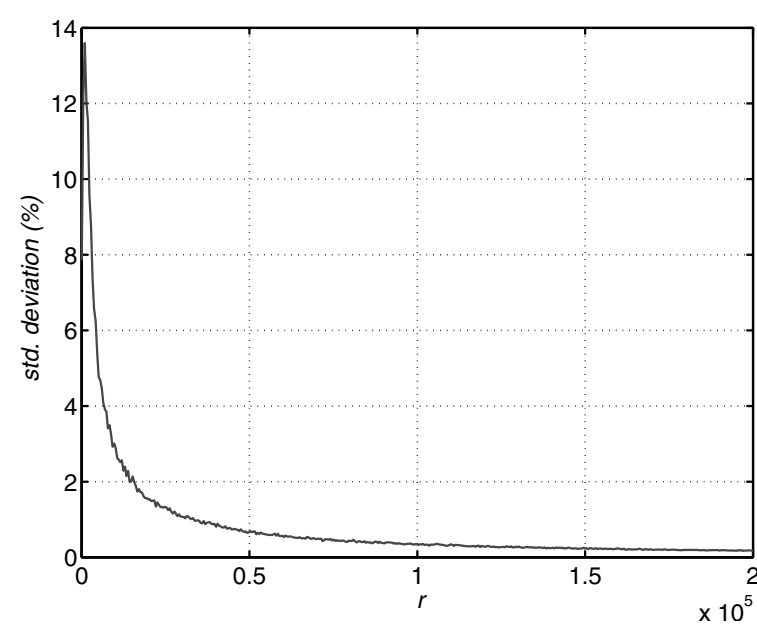

(b)

Fig. 2. Convergence plots of determinant bounds (max and min value for each iteration) (a) and the coefficient of variation (b).

Our goal is to identify the sources and the unknown diffusion coefficient, i.e., an estimate of the vector $\theta=\left(\mu_{1}, \chi_{1}^{1}, \chi_{2}^{1}, \mu_{2}, \chi_{1}^{2}, \chi_{2}^{2}, \kappa\right)$, using a sensor network with scanning nodes. The observation horizon was split into five evenly partitioned subintervals $T_{k}=(200(k-$ $1), 200 k], k=1, \ldots, 5$.

In order to verify the proposed approach, a MATLAB program was written using a PC equipped with an Intel Core i7 processor (1.83 GHz, 4 GB RAM) running Windows 7 and MATLAB 2009b. First, the system of PDEs was solved using efficient solvers of the COMSOL environment based on the finite element method (COMSOL AB, 2007). The nominal values of the system parameters were assumed to be $\theta=(12 \mathrm{~kg} / \mathrm{s}, 0.4 \mathrm{~km}, 0.7 \mathrm{~km}, 15 \mathrm{~kg} / \mathrm{s}, 0.8 \mathrm{~km}, 0.3 \mathrm{~km}$, $50 \mathrm{~m}^{2} \mathrm{~s}$ ). Calculations were performed for a spatial mesh composed of 978 triangles, 520 nodes and an evenly partitioned time interval (101 subintervals).

The observation grid was assumed to be created at locations selected from among those elements of the abovementioned 520-point triangulation mesh which do not lie on the outer boundary (there were 460 such nodes, which are indicated with dots in Fig. 3). Given $N=460$ prospective sites in $\Omega$, we aim at selecting at each time subinterval $T_{k}$ their subset consisting of the locations at which the measurements made by $n=100$ sensors would lead to D-optimum least-squares estimates of the parameters $\theta$.

It was assumed that the network was fully connected with uniform probability distribution for the connection between selected pair of nodes. The complexity of the system dynamics makes the proper prediction of the observation locations rather difficult and nonintuitive. The sensors tend to form patterns reflecting the areas of greatest changes in the pollutant concentration. Sensor configurations at different stages of the algorithm are shown in Fig. 3.
The convergence to the optimal sensor configuration is very fast at the first stage, which is clearly seen in Fig. 2(a). Since the algorithm deals with simultaneous optimization and the averaging of the FIM, we can observe that the variance of the distributed estimator increases at this phase (gap between bounds in Fig. 2(a)). Because of the increase in the total determinant value, a much better measure of the current variability of the averaging estimator is the coefficient of variation plotted in Fig. 2(b), where the convergence in the second moment is clearly illustrated.

After a relatively small configuration time $r$, the sensor activation pattern is very similar to the optimal one calculated with the use of centralized approach (cf. Figs. 3 3 c) and (d)). Then the algorithm considerably slows down and convergence with high accuracy requires many processes of pairwise communication.

\section{Conclusion}

The sensor scheduling problem in view of accurate parameter estimation for distributed-parameter systems subject to limitations on the total number of activated sensor nodes has been addressed. A crucial difficulty here is the large scale of the resulting global optimization problem, since the monitoring networks encountered in process industry or environmental engineering may often consist of several hundreds of nodes. This makes the exhaustive search on a candidate-by-candidate basis practically intractable and creates a strong necessity for more systematic and effective approaches. In particular, this work presents a proper adaptation of an approach based on the conversion of the original combinatorial optimization problem to its relaxed continuous approximation. The main idea is to operate on density of sensors, rather than on their direct 

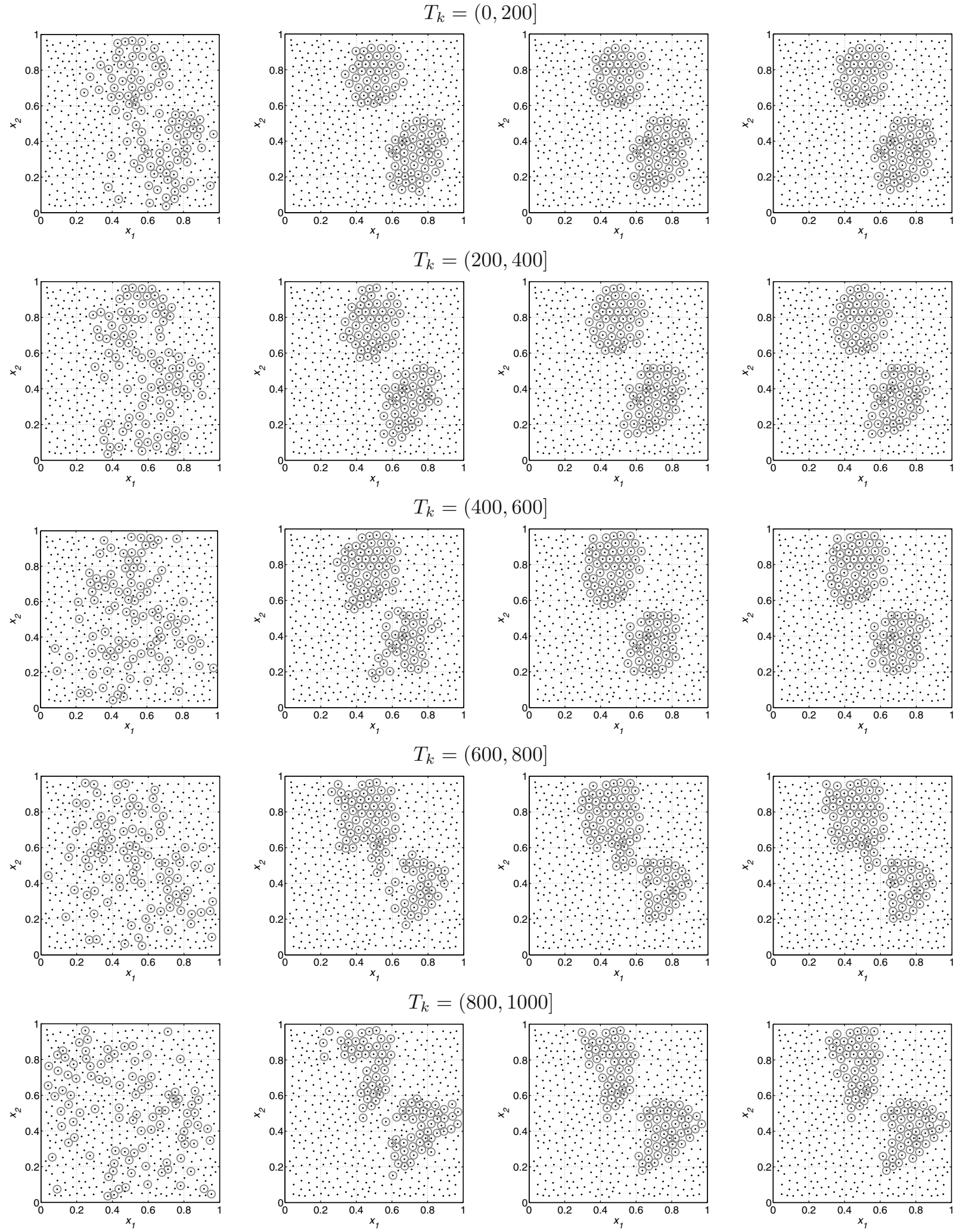

$$
\begin{gathered}
\mathcal{P}(\xi)=0.5537 \cdot 10^{12} \\
\text { (a) } r=460
\end{gathered}
$$

$$
\mathcal{P}(\xi)=1.5865 \cdot 10^{12}
$$

(b) $r=4600$

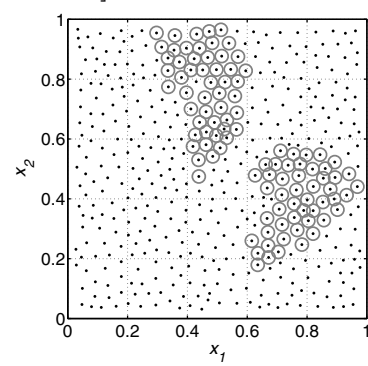

$\mathcal{P}(\xi)=1.7574 \cdot 10^{12}$

(c) $r=23000$

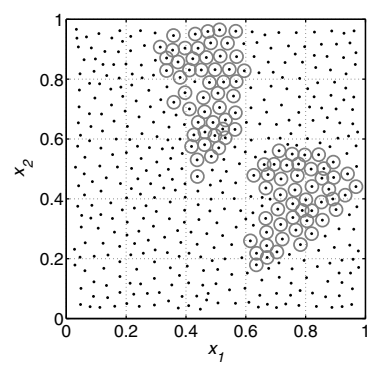

$\mathcal{P}(\xi)=1.7649 \cdot 10^{12}$

(d) D-optimal

Fig. 3. Allocation of active sensors at chosen observation subintervals at consecutive stages of network configuration (a)-(c) and final D-optimal configuration (d). 
locations, making it possible to apply some effective methods of convex optimization. Although such an approach is known in optimum experimental design theory, there have been just few attempts to exploit it in the context of decentralized computation schemes dedicated to sensor networks identifying DPSs (Patan, 2009b). This work contributes to this issue providing the proper mathematical formulation and characterization of the problem in the general setting of scanning observations.

Another crucial difficulty is a proper decomposition of the relaxed optimization problem in order to facilitate its efficient solution in a decentralized manner. The main contribution here consists in the construction of the specialized protocol of information exchange for the simultaneous optimization and estimation of the global optimality criterion at each node. Consequently, the proposed method leads to the fully distributed scheme of calculations which can be implemented with great ease, and our experience provides evidence that, with this tool, even large-scale design problems can be solved using an off-the-shelf PC.

Obviously, there still remain some open problems which require close attention. The following points can be raised as the main directions of further research:

- Extension of the discussed class of DPSs. A great advantage of the delineated approach is that it is independent of a specific form of PDEs used as a mathematical model of the DPS considered. In such a manner the presented approach can be rather easily adopted for the class of multi-output DPSs or/and systems with delays.

- Development of the approaches taking into account parametric uncertainty. A very important problem is the dependence of the solutions on the true values of the unknown parameters. This leads directly to the notion of the so-called robust design strategies (Sun, 1994; Uciński, 2005; Patan, 2004), which try to make the optimal solutions independent of the parameters to be identified.

- Extensions to more sophisticated monitoring systems, i.e., mobile sensor networks. In fact, a scanning network can be interpreted as mobile nodes whose positions are determined in discrete time.

- Refinements of the proposed approach taking into account numerous constraints inherent to network design, e.g., energy consumption or time-varying network topology.

\section{Acknowledgment}

This work was supported by the Polish Ministry of Science and Higher Education under Grant No. N N514 230537.

\section{References}

Amouroux, M. and Babary, J.P. (1988). Sensor and control location problems, in M.G. Singh (Ed.), Systems \& Control Encyclopedia. Theory, Technology, Applications, Vol. 6, Pergamon Press, Oxford, pp. 4238-4245.

Atkinson, A.C., Donev, A.N. and Tobias, R. (2007). Optimum Experimental Design, with SAS, Oxford University Press, Oxford.

Boyd, S., Ghosh, A., Prabhakar, B. and Shah, D. (2006). Randomized gossip algorithms, IEEE Transactions on Information Theory 52(6): 2508-2530.

Braca, P., Marano, S. and Matta, V. (2008). Enforcing consensus while monitoring the environment in wireless sensor network, IEEE Transactions on Signal Processing 56(7): 3375-3380.

Cassandras, C.G. and Li, W. (2005). Sensor networks and cooperative control, European Journal of Control 11(4-5): 436463.

COMSOL AB (2007). COMSOL Multiphysics Modelling Guide, ver. 3.4.

Demetriou, M.A. (2000). Activation policy of smart controllers for flexible structures with multiple actuator/sensor pairs, Proceedings of the 14th International Symposium on Mathematical Theory of Networks and Systems, Perpignan, France, (on CD-ROM).

Demetriou, M.A. and Hussein, I.I. (2009). Estimation of spatially distributed processes using mobile spatially distributed sensor network, SIAM Journal on Control and Optimization 48(1): 266-291.

Fedorov, V.V. and Hackl, P. (1997). Model-Oriented Design of Experiments, Lecture Notes in Statistics, Vol. 125, Springer-Verlag, New York, NY.

Jeremić, A. and Nehorai, A. (2000). Landmine detection and localization using chemical sensor array processing, IEEE Transactions on Signal Processing 48(5): 1295-1305.

Joshi, S. and Boyd, S. (2009). Sensor selection via convex optimization, IEEE Transactions on Signal Processing 57(2): 451-462.

Kempe, D., Dobra, A. and Gehrke, J. (2003). Gossip-based computation of aggregate information, Proceedings of the Conference on Foundations of Computer Science, Cambridge, MA, USA, pp. 482-491.

Kubrusly, C.S. and Malebranche, H. (1985). Sensors and controllers location in distributed systems-A survey, Automatica 21(2): 117-128.

Martínez, S. and Bullo, F. (2006). Optimal sensor placement and motion coordination for target tracking, Automatica 42(4): 661-668.

Müller, W.G. (2007). Collecting Spatial Data: Optimum Design of Experiments for Random Fields, 3rd Edn., PhysicaVerlag, Heidelberg.

Nehorai, A., Porat, B. and Paldi, E. (1995). Detection and localization of vapor-emitting sources, IEEE Transactions on Signal Processing 43(1): 243-253. 
Ögren, P., Fiorelli, E. and Leonard, N. E. (2004). Cooperative control of mobile sensor networks: Adaptive gradient climbing in a distributed environment, IEEE Transactions on Automatic Control 49(8): 1292-1302.

Patan, M. (2004). Optimal Observation Strategies for Parameter Estimation of Distributed Systems, Zielona Góra University Press, Zielona Góra, available at http: / /www. zbc. zgora.pl.

Patan, M. (2006). Optimal activation policies for continous scanning observations in parameter estimation of distributed systems, International Journal of Systems Science 37(11): 763-775.

Patan, M. (2008). A parallel sensor scheduling technique for fault detection in distributed parameter systems, in E. Luque, T. Margalef and D. Benítez (Eds.) Euro-Par 2008: Parallel Processing, Lecture Notes in Computer Science, Vol. 5168, Springer-Verlag, Berlin/Heidelberg, pp. 833843.

Patan, M. (2009a). Decentralized mobile sensor routing for parameter estimation of distributed systems, Proceedings of the 1st IFAC Workshop on Estimation and Control of $\mathrm{Ne}$ tworked Systems, NecSys 2009, Venice, Italy, pp. 210-215.

Patan, M. (2009b). Distributed configuration of sensor networks for parameter estimation in spatio-temporal systems, Proceedings of the European Control Conference, ECC'09, Budapest, Hungary, pp. 4871-4876.

Patan, M., Chen, Y. and Tricaud, C. (2008). Resourceconstrained sensor routing for parameter estimation of distributed systems, Proceedings of the 17th IFAC World Congress, Seoul, South Korea, (on DVD-ROM).

Patan, M. and Patan, K. (2005). Optimal observation strategies for model-based fault detection in distributed systems, International Journal of Control 78(18): 1497-1510.

Patan, M. and Uciński, D. (2005). Optimal activation strategy of discrete scanning sensors for fault detection in distributedparameter systems, Proceedings of the 16th IFAC World Congress, Prague, Czech Republic, (on CD-ROM).

Patan, M. and Uciński, D. (2008). Configuring a sensor network for fault detection in distributed parameter systems, International Journal of Applied Mathematics and Computer Science 18(4): 513-524, DOI: 10.2478/v10006-008-0045-4.

Patan, M. and Uciński, D. (2010a). Sensor scheduling with selection of input experimental conditions for identification of distributed systems, Methods and Models in Automation and Robotics, MMAR 2010: 15th International Conference, Międzyzdroje, Poland, pp. 148-153.

Patan, M. and Uciński, D. (2010b). Time-constrained sensor scheduling for parameter estimation of distributed systems, Proceedings of the 49th IEEE Conference on Decision and Control, Atlanta, GA, USA, pp. 7-12.

Point, N., Vande Wouwer, A. and Remy, M. (1996). Practical issues in distributed parameter estimation: Gradient computation and optimal experiment design, Control Engineering Practice 4(11): 1553-1562.
Porat, B. and Nehorai, A. (1996). Localizing vapor-emitting sources by moving sensors, IEEE Transactions on Signal Processing 44(4): 1018-1021.

Quereshi, Z.H., Ng, T.S. and Goodwin, G.C. (1980). Optimum experimental design for identification of distributed parameter systems, International Journal of Control 31(1): 2129.

Rafajłowicz, E. (1983). Optimal experiment design for identification of linear distributed-parameter systems: Frequency domain approach, IEEE Transactions on Automatic Control 28(7): 806-808.

Rafajłowicz, E. (1986). Optimum choice of moving sensor trajectories for distributed parameter system identification, International Journal of Control 43(5): 1441-1451.

Rao, M.M. (1987). Measure Theory and Integration, John Wiley \& Sons, New York, NY.

Song, Z., Chen, Y., Sastry, C. and Tas, N. (2009). Optimal Observation for Cyber-physical Systems: A FisherInformation-Matrix-Based Approach, Springer-Verlag, Berlin/Heidelberg.

Sun, N.-Z. (1994). Inverse Problems in Groundwater Modeling, Theory and Applications of Transport in Porous Media, Kluwer Academic Publishers, Dordrecht.

Uciński, D. (2000a). Optimal selection of measurement locations for parameter estimation in distributed processes, International Journal of Applied Mathematics and Computer Science 10(2): 357-379.

Uciński, D. (2000b). Optimal sensor location for parameter estimation of distributed processes, International Journal of Control 73(13): 1235-1248

Uciński, D. (2005). Optimal Measurement Methods for Distributed-Parameter System Identification, CRC Press, Boca Raton, FL.

Uciński, D. and Chen, Y. (2005). Time-optimal path planning of moving sensors for parameter estimation of distributed systems, Proceedings of the 44th IEEE Conference on Decision and Control/European Control Conference 2005, Seville, Spain, (on CD-ROM).

Uciński, D. and Demetriou, M. A. (2004). An approach to the optimal scanning measurement problem using optimum experimental design, Proceedings of the American Control Conference, Boston, MA, USA, (on CD-ROM).

Uciński, D. and Patan, M. (2002). Optimal location of discrete scanning sensors for parameter estimation of distributed systems, Proceedings of the 15th IFAC World Congress, Barcelona, Spain, (on CD-ROM).

Uciński, D. and Patan, M. (2007). D-optimal design of a monitoring network for parameter estimation of distributed systems, Journal of Global Optimization 39(2): 291-322.

Uciński, D. and Patan, M. (2010). Sensor network design for the estimation on spatially distributed processes, International Journal of Applied Mathematics and Computer Science 20(3): 459-481, DOI: 10.2478/v10006-010-0034-2.

van de Wal, M. and de Jager, B. (2001). A review of methods for input/output selection, Automatica 37(4): 487-510.

Xiao, L. and Boyd, S. (2004). Fast linear iterations for distributed averaging, Systems and Control Letters 53(1): 65-78. 


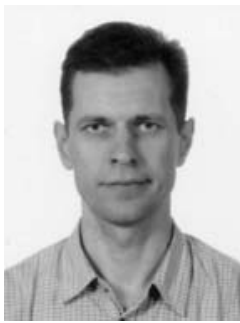

Maciej Patan was born in 1975. He received his M.Sc. and Ph.D. degrees, both in electrical engineering, from the University of Zielona Góra, Poland, in 2000 and 2004, respectively. Since 2004 he has been an assistant professor with the Faculty of Electrical Engineering, Computer Science and Telecommunications, University of Zielona Góra. From September 2005 to March 2006, he was a research assistant in statistics at the School of Mathematical Sciences, Queen Mary, University of London. He has been an IEEE member since 2009. His research interests include optimal experimental design in distributed parameter systems, distributed computing and optimization in sensor networks, system identification and mobile robotics.

Received: 12 February 2011

Revised: 30 September 2011 